Journal of The Electrochemical Society

jes.ecsdl.org.ezproxy.lib.utexas.edu

doi: $10.1149 / 2.005112$ jes

J. Electrochem. Soc. 2011 volume 158, issue 12, B1459-B1471

\title{
Manufacturing of Direct Methanol Fuel Cell Electrodes by Spraying
}

Babar M. Koraishy ${ }^{2}$ Jeremy P. Meyers" and Kristin L. Wood

\begin{abstract}
Spraying is a well-established coating process used to fabricate electrodes for both polymer electrolyte membrane fuel cell (PEMFC) and direct methanol fuel cells (DMFCs), and also for the fabrication of gas-diffusion media (GDM) used in fuel cells. Despite its popularity as a process there is little basic research on how spray parameters and nozzle characteristics affect the droplet sizes of catalyst inks, and how the droplet sizes affect the electrode structure, and eventually the overall membrane-electrode assembly (MEA) performance. We present results from an experimental study to quantify key process parameters in the electrode spraying process, characterizing the spray nozzle, measuring droplet diameters, and investigating the microstructural effects on electrode performance. For this purpose, a spraying apparatus was developed and calibrated, and MEA's were fabricated with fixed electrode loadings but with different droplet sizes. Droplet sizes were controlled by characterizing the spray nozzle and measuring the spray optically by utilizing high-speed photography. It is shown that increasing spraying pressures generally reduces the mean droplet size of the spray, which affects the microstructure of the electrode produced and results in higher MEA performance.
\end{abstract}

Of the costs involved in hydrogen-based PEMFCs and DMFCs, the material cost of the catalyst and the ion-exchange membrane are key contributors to the overall system cost, but even if much less expensive materials were available, the labor-intensive nature of the membrane electrode assembly (MEA) processing contributes a major portion of the manufacturing costs, particularly at low production volumes. ${ }^{1}$ Performance issues, which range from overall system energy density, to long-term performance and stability, are likewise tied directly to the materials and the manufacturing process choices.

The porous electrode in the MEA is formed by depositing catalyst ink onto a substrate, which, upon drying, forms a three-dimensional porous structure, which allows for the transport of the fuel and oxidant to the electrode and for the removal of reaction products from the electro catalysts. The MEA electrode must be electronically conductive to allow for the movement of the electrons to and from the external circuit, and must maintain an ionic pathway for the protons to move through the ionomer to the other electrode to complete the overall reaction. This process is shown schematically in Figure 1. Limiting the mobility and transport of any of these species reduces the catalyst utilization and the overall performance that can be extracted from a given amount of catalyst.

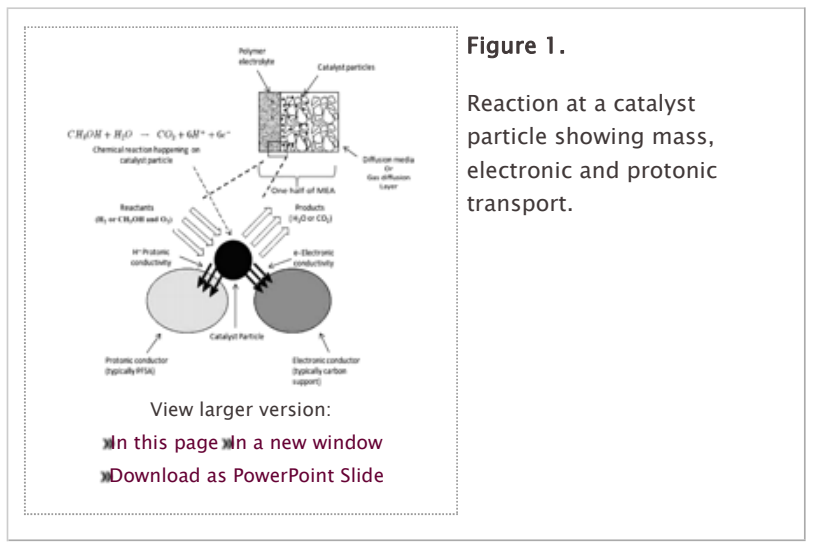

From a performance standpoint, it is important to study the ink application process choices and process parameters in detail, and understand how they can 
be related to quantifiable and measurable fuel cell performance metrics. It is essential to engineer the electrode for maximum mass transport and minimal loading of the expensive catalyst material.

The catalyst ink application onto the surface of a substrate is a required step for electrode fabrication, irrespective of the type of MEA being fabricated. In the ink application step, the catalyst ink is distributed uniformly onto the surface of the substrate. It is important that catalyst loading is maintained at a uniform level across the width and length of the electrode. The microstructure of the electrode formed during the ink application and drying stage is critical. As a result, it is important to avoid agglomeration of the catalyst particles, or of the associated binder, so as to ensure an electrode where the catalyst is thoroughly dispersed, and the triple phase boundary is maximized. This will ensure high catalyst utilization. A thorough review of the relevant manufacturing processes for fuel cells is provided by Mehta et al. ${ }^{2}$

Spraying is an established coating process used for the application of catalyst ink on a desired substrate. ${ }^{3-9}$ In the context of catalyst ink spraying, the atomization process involves the decomposition of the catalyst ink suspension into droplets upon entering, mixing, and interacting with air at a nozzle orifice. The catalyst ink is provided sufficient energy to penetrate the ambient fluid and facilitate subsequent dispersion by a separate stream of high-pressure air. This energy can be provided by the kinetic energy of the liquid that is to be atomized, by the action of high velocity gas, or by mechanical energy applied through an external device. 10 Such devices, which are used to produce the droplets, are called atomizers.

There have been other papers written on using the spraying technique to create high-performance catalyst layers in fuel cell applications in both PEM and other fuel cell configurations, but most papers have focused on influence of composition and catalyst loading, rather than a detailed manufacturing study ${ }^{11,12}$ Despite the appeal of spraying as a catalyst layer manufacturing technique, there is little basic research on how spray parameters and nozzle characteristics affect the droplet sizes of catalyst inks, and how these droplet sizes affect the electrode structure, and eventually the overall MEA performance.

\section{Background}

Atomizers

Atomizers are classified by the type of energy employed to produce perturbations and instability in a fluid. These instabilities lead to the detachment of smaller volumes from the bulk fluid, resulting in droplet formation. A nozzle is an atomizing device that ejects a liquid through an orifice. Other types of atomizers are centrifugal devices that spin and thereby create droplets, electrostatic atomizers, and ultrasonic vibrators.

Table 1 summarizes the key characteristics of the various atomizing processes in the context of the current application, which requires spraying viscous ink onto a substrate at close proximity. Processes such as pressure atomization and rotary atomization are not considered because of the high atomizing pressure and fluid flow rate requirements, as well as the geometry of the spray produced. Higher pressures impart significant energy to the spray, which can result in loss of catalyst ink. Two other processes, ultrasonic and electrostatic atomization, are very attractive, as the fluid atomization does not involve pressurized air, but the limitations are that they typically operate at very low flow rates, and the atomized fluid must be of low viscosity. Based on these considerations, it is concluded that the two-fluid, external mixing, air-assist type nozzle is a suitable choice for the MEA manufacturing process, and the focus of the study reported here.

\begin{tabular}{|c|c|}
\hline $\begin{array}{l}\text { View this table: } \\
\text { wn this window wn a new window }\end{array}$ & Table I. \\
\hline praying catalyst inks on a substra & $, 13,28$ \\
\hline
\end{tabular}

In an air-assist atomizer, the atomization occurs due to the kinetic energy of a flowing air stream, which causes the liquid stream to break-up into fine droplets. Air-assist atomizers typically operate at low airflow rates, but there 
are no restrictions on air pressure and air velocity, which are chosen according to the fluid to be atomized. They are well suited for atomizing highly viscous fluids. ${ }^{10}$ Air-assist atomization is compatible with viscous fluid flow and can easily produce solid cone, hollow cone, and flat fan spray patterns. Air-assist nozzle generally require high air pressures to effectively atomize fluids, but low -volume, low-pressure atomizers such as the Nordson EFD 781S do exist, and are used for the application of paints and coatings. ${ }^{13}$

Droplet size measurement

A spray is characterized on the basis of its shape, patternation, and droplet size. The nozzle orifice controls the shape and patternation of the spray. Patternation is the flux of droplets across a plane in the spray pattern; it is the spatial distribution of droplets within the spray. It refers to both the distribution and the shape of the spray. The other property of interest is the droplet size produced by this nozzle. The next sections discuss the significance of droplet size, and the apparatus used to perform droplet size measurements.

The controllable parameters of a two-fluid, external mixing, air-assist type nozzle are the air pressure, the fluid pressure, and the effective nozzle area. Other parameters that could potentially affect the mean droplet size of the spray include the nozzle orifice diameter and ink viscosity. It has been observed that different combinations of these parameters produce sprays with different characteristics, which, from visual inspection, could be identified as ranging from 'coarse' to 'fine'. We therefore seek to characterize and compare inks generated with different spraying conditions, determine the effect of sprays on the microstructure of the electrode produced, and determine the optimal settings for the spray nozzle in terms of manufacturability and performance in a fuel cell. As such, we began with an investigation of spraying processes to characterize the sprays that can be produced by the nozzle, with particular emphasis on the mean droplet size.14-18

\section{Droplet size measurement techniques}

Measuring droplet size is a challenging task, due to the vast number of droplets in a spray and the wide range of velocities and sizes. Ideally, nonintrusive or minimally intrusive processes are preferred to prevent altering or disturbing the atomization process or spray. It is likewise important to obtain a large sample size to accurately represent the droplet size distribution within a spray. ${ }^{10}$ Numerous drop size measurement techniques are available, and can be grouped into a four broad categories: mechanical, electrical, optical, and acoustic techniques.

Mechanical methods involve the physical capture of droplets on a media such as a slide, after which the drops are individually measured and counted. Concerns with this method are that the drops flatten upon impact and, as a result, correction factors must be used. Very small droplets generated in the spraying process are also susceptible to effects of evaporation, which potentially produces erroneous measurement results. 10

In electrical methods, both charged-wire and hot-wire techniques measure the impact of a droplet on a wire. In the charged wire technique, a change in the charge is measured. In the hot wire technique, local cooling of the wire causes a change in resistivity, which is measured. Both electrical methods are intrusive. The charged wire method performs well only with high conductivity fluids, and the hot wire method is limited to fluids that will not leave a residue on the wire. ${ }^{19}$

High-speed photography is considered to be one of the least expensive and most accurate droplet sizing methods available. Associated challenges are accurately focusing the optics and counting the droplets that are captured in an image. 10 Based on accuracy, ease of use, cost, and availability, high-speed photography and the hot wire technique were selected as part of this study.

\section{Experimental Approach}

Droplet size measurement: Hot-wire technique

In the hot-wire technique, a hot-wire anemometer is used to measure the velocity and droplet size distribution of a spray. It is an intrusive technique in which a heated, $5 \mu \mathrm{m}$ diameter platinum wire is introduced into the spray. When a droplet impinges on this heated wire, it reduces the resistance of the wire upon evaporation. This change in resistance is in proportion to the size of 
the droplet. Each individual measurement takes a few milliseconds, prescribing the overall data sampling time needed to collect sample points for an accurate representation of the size distribution in the spray. From this data, the mean droplet size and spray velocity are calculated.

A KLD labs DC-III hot wire droplet analyzer20 was used in the drop size and velocity experiments. Operation of the device is a fairly simple procedure: the analyzer module is connected to a computer, the accompanying software initiates a self-test and calibration procedure, sampling time interval and fluid type are selected, and the probe is positioned in the spray. Upon completion of the data collection, spray distribution and spray statistics are presented.

The hot-wire method relies on the evaporation of the drop on the wire. Catalyst inks, however, contain solids and particulate matter. When using catalyst inks, upon evaporation of the solvents, a solid residue remains. Accumulation of this residue on the wire prevents subsequent droplets from directly contacting the wire surface, which thereby limits the duration of experiment before the wire must be refreshed. This accumulation of material further disrupts the calibration of the instrument, as the algorithms for calculating the sizes are specific to the fluid used, which in the case of the DC-III analyzer, were limited to water and mineral oils.

Another complication of using a hot wire anemometer is the delicate nature of the probe used in the measurement. The probe contains a fine, platinum wire 5 $\mu \mathrm{m}$ in diameter, which must be placed in the spray. This wire is extremely delicate and prone to breakage due to the impact of droplets and handling. It also has to be carefully cleaned in solvents prior to each use, to remove any residue that might have accumulated on the wire surface.

Due to the reasons mentioned, it was difficult to use the hot wire technique directly with catalyst inks. Instead, it was used for two very important and alternative purposes. The first was that the DC-III droplet analyzer was used to probe and identify the velocity of the droplets at different distances from the nozzle. This data was needed to identify the height at which the high-speed camera should be placed for the high-speed photography based droplet size measurement system. Additionally, it was used to compare results of an atomized water spray with the photography method, details of which are described in the following sections.

\section{Optical method: High-speed photography}

There exists a multitude of droplet size measurement technologies based on optical recognition of a droplet, but high-speed photography offers a significant cost advantage and is considered to be a very accurate method for droplet size measurements.

The goal in high-speed photography is to 'freeze' the motion of a fast-moving object, a droplet in this case, and capture it on a suitable media. For the purpose of photographing the atomized spray of catalyst inks used in the manufacture of PEMFC and DMFC fuel cells, we selected a method with a high camera shutter speed and constant background lighting, as it was less costly to source a camera with a fast shutter, while maintaining measurement accuracy, than to procure a high-speed flash. As shown in Figure 2, the overall imaging system consists of a constant light source, a camera with an extremely fast shutter, and the necessary optics to magnify the droplets so that they can be successfully measured.

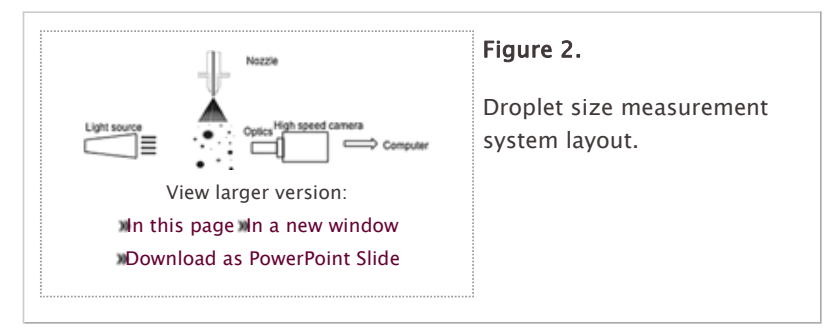

The first step in the measurement process is to capture an image. This is achieved by the optical system, comprised of a high-speed CCD camera (AMAZON IMB-7015G 1/3 CCD $1296 \times 964$ pixels), and a 5W LED spot light (Advanced Illumination SL-073). The maximum frame rate of the camera is 30FPS, but the more important parameter is the minimum shutter speed it can 
achieve: $1 \mu \mathrm{s}$, an appropriate shutter speed to photograph the relatively low velocity droplets $(\sim 8 \mathrm{~m} / \mathrm{s})$ being studied. The camera communicates with a host computer using a gigabit Ethernet connection to ensure fast data transfer and maximum compatibility.

Two interchangeable lens systems were mounted on the camera. The first was for large droplet sizes: a 12.5-75 mm Navitar TV zoom lens was used in conjunction with $35 \mathrm{~mm}$ spacers. The second setup, as shown in Figure 3 , involved using a 10X long working distance Mitiyoto microscope objective mounted on a lens tube with $35 \mathrm{~mm}$ spacers. This setup provided for higher resolution to capture smaller droplet sizes.

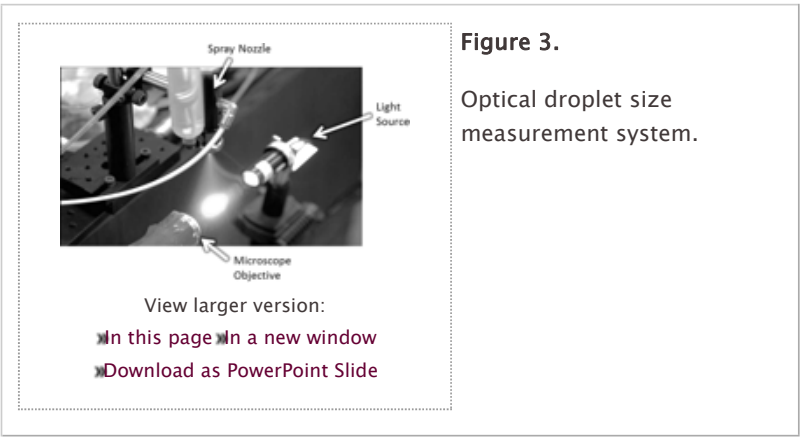

Once the image is captured, the data must be analyzed. Digital image capture and advanced digital image processing algorithms allow image processing to be completed automatically by software.10,15 Irrespective of the sizing method used, the net result is a distribution of droplet sizes, from which further calculation produces the length mean diameter (LMD). This number is the arithmetic mean of the droplet size distribution and is useful in droplet size comparison of two sprays. Other 'diameters,' such as the surface mean, volume mean, and Sauter mean diameter (SMD), represent the mean surface area, mean volume and volume to surface area ratio of sprays.21 The choice of diameter is dependent upon which spray characteristic is of interest. In the case of catalyst ink sprays, the size of different particles is being studied, so the length mean diameter is most relevant.

In order to process the image data, ImageWarp, an image-processing program from ab-soft.com, aided in automating the process.22 ImageWarp contained prebuilt image processing functions that were pieced together to create a script that detected the edges of droplets within the captured images, processed them, and output the size of each droplet measured. The first step in processing the image was to apply a median filter on the image, which is a popular technique in digital image processing to remove noise from an image. This step was carried out to remove any erroneous geometric artifacts present in the original image. The second step was to perform edge detection on the image, as shown in Figure 4. This step is carried out by identifying closed contours in the image and then separating them from the background. The ImageWarp software came with prebuilt functions that performed these actions. Once these edges are isolated, they are filled with an area and then classified according to their size. The final step was to display the detected drops as an overlay on the original image and output a distribution of the diameter of drops detected in the image. Hundreds of images were processed to produce a droplet size distribution, which consists of at least 5000 data points. This data size is required for an adequate representation of the spray. 10

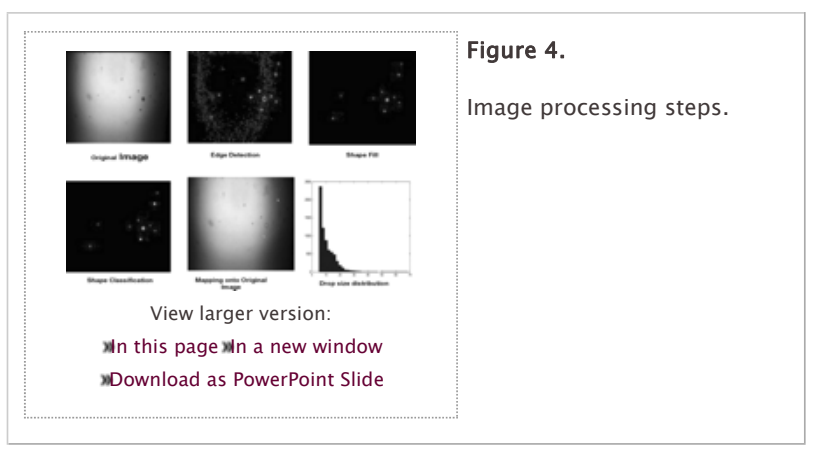

http://jes.ecsdl.org.ezproxy.lib.utexas.edu/content/158/12/B1459.full?sid=2b9e4079-8830-4... 8/9/2012 


\section{Surrogate inks for droplet size measurement experiments}

The next stage in the nozzle characterization process was to study the spray characteristics of the nozzle with various fluids: water and catalyst inks of various solid contents. The aim behind these experiments was to identify the relationship between droplet sizes, air pressure and flow rate. Fluids of different viscosities were studied so that the impact of changing viscosity on the drop size could also be identified.

The volume of catalyst ink required to perform these experiments would be substantial, and a tremendously large amount of platinum catalyst would have to be used to produce these inks. A significant quantity of these ink solutions would ultimately go to waste once they have been sprayed from the nozzle. It was decided to design surrogate inks, which would emulate the performance of catalyst inks.

Catalyst inks used in PEMFC and DMFC manufacture can contain supported or unsupported nanometer-sized noble metal particles.9,23 Supported catalysts are essentially larger carbon particles onto which the catalyst particles are attached. This allows for better dispersion and utilization of catalyst in the electrode layers. Supported catalysts range from $20 \%$ to $80 \%$ noble-metal loading, where $\%$ noble-metal loading corresponds simply to the carbon black support material and $100 \%$ noble-metal loading corresponds to unsupported, pure catalyst black. Other constituents of the catalyst inks are a suitable amount or ratio of ionomer and solvents, typically water and Isopropyl alcohol (IPA). Bearing this information in mind, it was determined that ink with only carbon black solid contents, along with the necessary ionomer and solvents would represent the characteristics of catalyst inks used in PEMFC and DMFC, and could be used to emulate its behavior during spraying.

A variety of carbon inks were created, containing between $2 \%$ and $10 \%$ suspended carbon and Nafion ${ }^{1}$ solid content, and the viscosities of these inks were studied. The purpose of the carbon ink viscosity experiment was to determine a correlation between the ink solid content and its viscosity; the utility of this data is that it could be used to predict the behavior of actual catalyst inks of different solid contents. The viscosity instrument used was a Brookfield DV-E Viscosity Meter with a number 18 spindle. Because viscosity is a strong function of temperature, a constant temperature of $22^{\circ} \mathrm{C}$ was maintained. ${ }^{24}$ The actual viscosity measurements for the produced surrogate inks are presented in detail in the subsequent section on fabrication and testing. The surrogate catalyst ink behaves as a Non-Newtonian fluid, due to the solid and polymer content. The shear forces acting on the ink as the it exits the nozzle tend to assist in droplet formation, but a detailed study of the fluid mechanics in this system has not been attempted here. As such, it is not clear how the non-Newtonian nature of the ink will affect the optimum set of control parameters, beyond the results presented in the results section.

\section{MEA fabrication and testing}

DMFC MEA fabrication by the decal-transfer method consists of a four steps: membrane preparation, electrode fabrication, electrode transfer, and finally the attachment of diffusion media on the 3 layer MEA produced, to form a 5-layer MEA.

Nafion -115 membranes were prepared to receive the catalyst ink. First, the membrane was cleaned to remove any unwanted substances. The cleaning process involved boiling the membrane in $3 \mathrm{wt}$ \% hydrogen peroxide $\left(\mathrm{H}_{2} \mathrm{O}_{2}\right)$ for $1 \mathrm{~h}$ and then boiling it in deionized water for $1 \mathrm{~h}$. The membranes were boiled in $1 \mathrm{M} \mathrm{H}_{2} \mathrm{SO}_{4}$ for $1 \mathrm{~h}$ and then boiled in deionized water for $1 \mathrm{~h}$, as per the procedure defined by Ren. 25 Catalyst inks for the DMFC anode were prepared using unsupported Pt:Ru (1:1) HISPECTM 6000, platinum procured from Alfa Aesar; Pt Black (high surface area), also procured from Alfa Aesar, was used as the cathode catalyst material. An equal ratio (1:1) mixture of deionized water and isopropyl alcohol (IPA) was used as a solvent in the ink. A $5 \%$ solution of Nafion was used as the binder.

Catalyst particles have a tendency to ignite if directly exposed to IPA vapor therefore, ink preparation began with a mixture of water and the as-received Nafion solution, to which small quantities of catalyst particles were subsequently added added. Each step in the mixing process was accompanied by thorough ultrasonic mixing. We found it necessary to add the catalyst black in small quantities and thoroughly mixing at each step to form a uniform 
suspension, because the catalyst black particles have a tendency to form agglomerates. If the entire amount of catalyst were to be added in one step, the ink suspension formed would not homogenous. Once all the catalyst has been suspended into the water and Nafion solution, then only is the required quantity of IPA added. The surrogate inks used in the experiments were produced by the exact same procedure, but must we found that forming a suspension of carbon black particles was significantly easier and could accommodate larger stepwise additions of solids, as compared to catalyst black particles.

The next step in the electrode fabrication involved the application of catalyst ink onto a suitable substrate. Kapton tokens were mounted onto a vacuum table that was maintained at a constant temperature of $60^{\circ} \mathrm{C} .{ }^{23} \mathrm{~A}$ mask was affixed onto the vacuum plate, which included a cut-out void area of the desired electrode size. This allowed for clean edges of the electrode, and ensured that the catalyst ink is only deposited in the desired area. Spraying was performed by using a two-fluid external mixing Nordson EFD-781S nozzle, which was mounted in a purpose built 2 -axis computer-controlled machine, as shown in Figure 5. This apparatus provided a mounting platform for the nozzle and corresponding armature to allow for fine-tuning of the nozzle's aim, in addition to adjustment of the working distance of the nozzle from the material. As shown in the figure, the spraying nozzle was positioned above a heated vacuum mounting plate. The nozzle was driven by a Nordson EFD ValveMate 8040 controller, which permitted for the triggering of the nozzle, when instructed by a host computer. The host computer controlled the motion of the nozzle head mounted on the $X-Y$ table and synchronized it with the nozzle triggering at the desired locations. In this fashion the desired geometry of the electrode was sprayed uniformly onto the substrate.

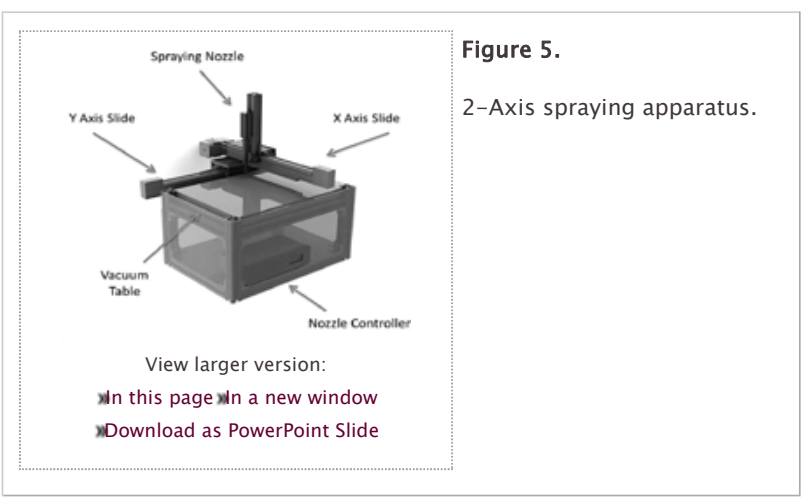

For the decal-transfer process, a low-temperature, high-pressure process was chosen; the transfer temperature was set at $130^{\circ} \mathrm{C}$ while the pressure used was $5000 \mathrm{psi}$ for $180 \mathrm{~s}$ duration. The last step of the electrode fabrication procedure is to attach the diffusion media to each electrode. Two appropriately sized sections of diffusion media (ELAT LT-1400, ETEK) were affixed onto the 3 -layer MEA formed with the assistance of temperature and pressure. A temperature of $120^{\circ} \mathrm{C}$ and a pressure of 1200 psi was used for this step for a $60 \mathrm{~s}$ duration as described in. 26

A Fuel Cell Technologies test station was used to test the fabricated MEAs, along with single-cell $5 \mathrm{~cm}^{2}$ hardware provided by the same company. A new MEA was first subjected to a 24 hour break-in period. The break-in procedure involved cell installation in single-cell hardware with an axial load specified by a bolt torque set at $40 \mathrm{in}-\mathrm{lb}$., followed by leak testing. For testing, the backpressure was then set at $20 \mathrm{psi}$ and the cell temperature was maintained at $90^{\circ} \mathrm{C}$. Dry oxygen was supplied to the cathode at $250 \mathrm{SCCM}$, and $1 \mathrm{M}$ aqueous methanol solution was supplied to the anode compartment at $2 \mathrm{ml} / \mathrm{min}$. The cell was maintained at $0.2 \mathrm{~V}$ for 24 hours. After the break-in period, the MEA was tested in conditions summarized in Table 2.

\begin{tabular}{|c|l|}
\hline View this table: & Table II. \\
\hline mn this windowmn a new window & Testing conditions for $5 \mathrm{~cm}^{2}$ \\
& single cell DMFC MEA. \\
\hline
\end{tabular}

http://jes.ecsdl.org.ezproxy.lib.utexas.edu/content/158/12/B1459.full?sid=2b9e4079-8830-4... 8/9/2012 


\section{Results}

\section{Drop velocity measurements}

Figure 6 shows how the bulk velocity of droplets produced by the Nordson EFD 7815 nozzle changes as a function of the distance from the nozzle. Initially the drop velocity is very high, but the drops decelerate due to aerodynamic drag and reach their terminal velocity, as shown in measurements taken at longer distances from the nozzle. Since water was used to measure the velocity, the calibrations in the instrument were not disturbed. The water velocity data was used as a guide to determine the camera distance for the ink experiments.

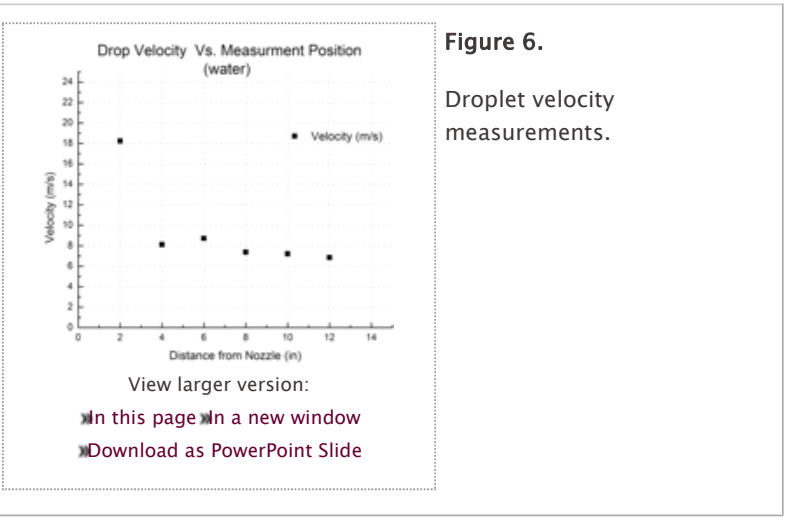

As shown in the Figure, beyond a distance of 4 inches from the nozzle, the drop velocity remains constant. We attribute this to the droplet reaching its terminal velocity. The influence of atomizing air and fluid pressure is what imparts kinetic energy to the droplet in the first place, after which the drop decelerates due to aerodynamic drag, until it reaches its terminal velocity. The inks used in this study have a solid content of $4 \%$, the balance of which is made up of water and alcohol. Since the droplet diameter is much larger than the typical diameter of carbon particles in the ink, we assume that the solid particles are encompassed by fluid, and therefore the surrogate ink droplet as it moves through the air will undergo similar aerodynamic forces as would a water droplet, and would follow the same general trend of the velocity profile as shown in Figure 6. There are, of course, differences in effective friction factors between pure liquid droplets and droplets with solids at their core, but we considered that most of the differences would be inertial in nature, so the terminal velocities would be similar. The assumption that the catalyst ink would follow similar deceleration as a water drop was verified, as there was no evidence of streaking in the catalyst ink photographs. These streaks would have been present if the drop was moving too fast.

\section{Surrogate ink viscosity measurements}

Figure 7 shows how the ink viscosity increases nonlinearly as the overall solid content of the ink solution is increased. The general nonlinear nature of this relationship is expected, where the trend can be attributed to the increasing polymer (Nafion) content. Increasing the polymer content in the ink increases the content of insoluble solids in the ink slurry, which contributes significantly to the viscosity of that ink. This data served to guide our understanding as to how the nozzle spray characteristics change with varying solid content of the inks, in order to tailor the manufacturing process for inks of various solid contents. Controlling the spray characteristics was therefore necessary to regulate the amount of catalyst per sprayed layer, potentially to reduce the requisite number of layers to achieve a specific electrode loading. Increasing air pressure imparts greater kinetic energy to the droplets. It is believed that this higher energy not only flattens, but also actually breaks the droplet upon impact onto the platinum wire in the hot wire method. This would result in lower LMD values as reported by the hot wire apparatus. Such a trend is clearly visible in the data shown in Figure 8, where the hot wire method consistently reports lower LMD values as compared to the optical method.

Figure 7.

Carbon ink viscosity vs. Ink solid content. 


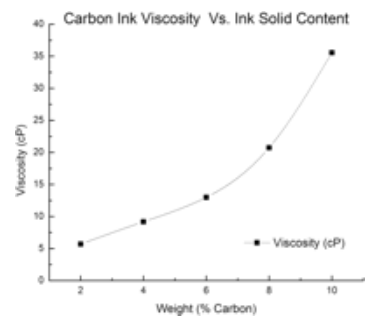

View larger version:

Wn this page $\mathrm{m}$ a new window

wDownload as PowerPoint Slide

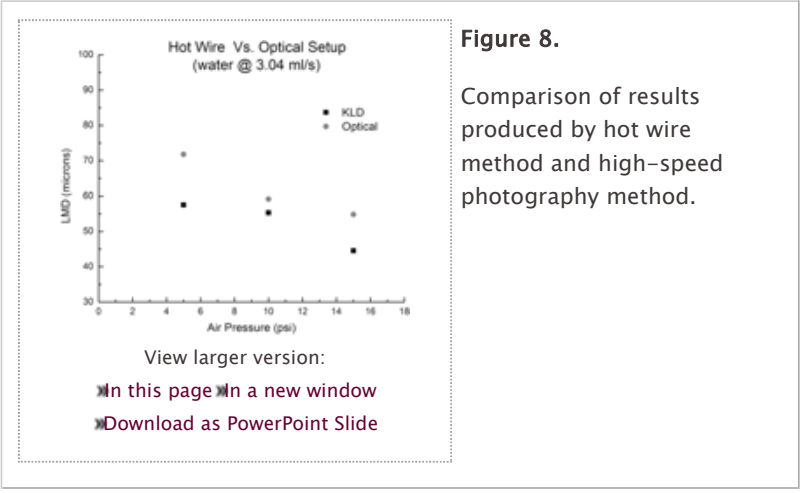

Comparison of droplet size measurement results obtained by hot-wire method and optical method

As described earlier, the hot wire method's applicability was extremely limited due to the fact that it was incompatible with catalyst inks, as the solids contained in the ink would deposit on the platinum wire, thereby limiting the accuracy and efficacy of the data measurements. This method does contribute, however, to the ability to corroborate the results of the high-speed photography apparatus by comparing results of each method, using water as the medium. Figure 8 shows the length mean diameter (LMD) results of a spray produced by the Nordson EFD-781S nozzle at various pressures, measured by both apparatuses, using water as the fluid. The figure indicates that there is an adequate correlation between the data. The results produced by the hot wire anemometer are consistently lower than that of the high-speed photography setup, an observation that has also been noted in prior studies comparing different droplet-sizing instruments. ${ }^{14}$

\section{Nozzle characterization}

The purpose of this experimental development is to create and validate the tools that will allow us to study and characterize the effects of changing the control parameters of a nozzle. To this end, a high-speed photography setup was devised and calibrated to measure the droplet diameters of the spray produced, as discussed above.

For a two-fluid external mixing type air-assist nozzle, there are three control parameters of interest: the atomizing air pressure, fluid pressure, and plunger setting. The "plunger setting" refers to how a plunger needle is used to meter the fluid flow rate of the atomizing nozzle. In order to characterize the nozzle, the first step is to control the flow rate of the fluid that is being atomized. One would infer that, ideally, the flow rate would be a function of the nozzle orifice area through which the fluid is discharged, as controlled by the plunger setting, and the fluid pressure; however, these experiments used an external mixing nozzle, and the fluid exits the nozzle orifice in the midst of a stream of pressurized air. Therefore, there might exist interactions between the fluid flow rate and the atomizing air pressure, which could influence the flow rate of the fluid. The first section below studies this interaction and presents an empirical model for the flow rate of this nozzle. A subsequent section presents empirical models for three different fluids used as media for the nozzle in question. These models facilitate identification of the different droplet sizes possible at different control parameter settings.

http://jes.ecsdl.org.ezproxy.lib.utexas.edu/content/158/12/B1459.full?sid=2b9e4079-8830-4... 8/9/2012 
Flow rate model

In the context of spraying catalyst inks onto a substrate, the flow rate and the velocity of the spray nozzle relative to the substrate, whether with a moving substrate and fixed nozzle or a moving nozzle and stationary substrate, determines the wet layer thickness. The wet layer thickness, in turn, defines the catalyst loading on the electrode. The amount of ink that is deposited onto the substrate must be subsequently dried; therefore, it is important to accurately control the fluid flow rate.

A design of experiments (DOE) approach was undertaken to identify possible coupled effects between the different control variables and the response of the resulting layer. 27 We considered three control parameters: air pressure, fluid pressure, and plunger setting. The "plunger setting" refers to how a plunger needle is used to meter the fluid flow rate. This is achieved by the insertion or removal of the plunger needle in the fluid orifice, which effectively changes the area through which fluid can exit. An experiment was designed in which these parameters were varied systematically to determine the flow rate response. A $2^{k}$ factorial design was selected, where $k$ factors (fluid pressure, area, and air pressure) were run at 2 levels each (high and low, represented by "+" and "-" where the signal response, i.e., flow rates, were measured at these settings. Table 3 shows the experimental design used, following Yates' standard form.

\begin{tabular}{|c|l|}
\hline View this table: & Table III. \\
\hline mn this window wn a new window & Experimental design for $2^{3}$ \\
& flow rate model. \\
& \\
\hline
\end{tabular}

In the present case, $k=3$, as there are three factors. The general bi-linear regression model used to fit this data is:

$$
\begin{array}{r}
y=\beta_{0}+\beta_{1} x_{1}+\beta_{2} x_{2}+\beta_{3} x_{3}+\beta_{4} x_{1} x_{2} \\
+\beta_{5} x_{1} x_{3}+\beta_{6} x_{2} x_{3}+\beta_{7} x_{1} x_{2} x_{3}
\end{array}
$$

Where $x_{1}, x_{2}, x_{3}$, represent the model variables and $\beta_{o} \beta_{7}$ represent the regression coefficients that were calculated during model fitting. Four replicates were run for each setting for a total of 32 observations. Results of the bi-linear regression model for the flow rate data are visually presented in Figure 9. The volumetric flow rate of water was calculated for three different pressure settings and three different plunger settings, which represents the open area of the fluid orifice. As shown, the flow rate increases with increasing fluid pressure and with an increasing value of the plunger setting (fluid orifice area).

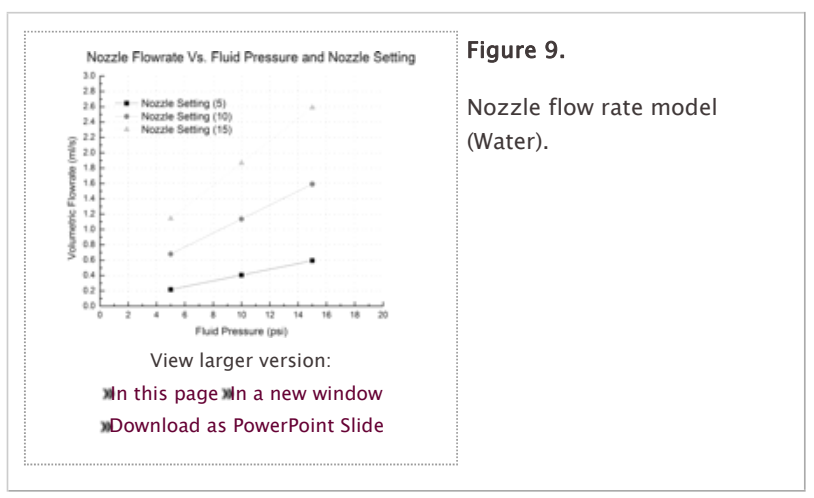

Droplet size models

Having reduced the three control parameters of the nozzle into two variables, namely the flow rate and atomizing air pressure, a study was conducted to ascertain how the spray characteristics change with these variables. The length mean diameter $(\angle M D)$ is used to reduce the droplet size distribution obtained into a single number, so that a spray produced at one setting can be compared with another produced at a different setting.

An experiment was designed and a second order model was fit to the resulting data to create a model to predict the $\angle M D$ of a spray as a function of air 
pressure and fluid flow rate. A $3^{k}$ design was used (where $k=2$ ) with two replicates for a total of 18 readings. Three fluids were characterized: water, $2 \%$ solid content ink, and $4 \%$ solid content ink. The general form of the second order regression model used is: 27

$$
y=\beta_{0}+\beta_{1} x_{1}+\beta_{2} x_{2}+\beta_{3} x_{1} x_{2}+\beta_{4} x_{1}^{2}+\beta_{5} x_{2}^{2}
$$

Table 4 shows the experimental design used. The 2 factors in the experiment (flow rate and air pressure) were run at three different levels (low, medium and high, depicted by "-", "0", and "+", respectively). The response $(L M D)$ was measured by high-speed photography, as described previously.

\begin{tabular}{|c|l}
\hline $\begin{array}{c}\text { View this table: } \\
\text { wn this window wn a new window }\end{array}$ & Table IV. \\
& $\begin{array}{l}\text { Experimental design for } 32 \\
\text { droplet size }(L M D) \text { model. }\end{array}$
\end{tabular}

Analysis of droplet size models

Figure 10 shows the dependence of the LMD on the two control variables, air pressure and fluid flow rate. There are three surfaces shown in the plot. The top surface represents water, the middle represents a $2 \%$ solid content ink, and the bottom surface represents a $4 \%$ solid content ink. A clear trend is clearly evident by comparing these three surfaces. As the viscosity of the fluid increases, the LMD values are lower for the same air pressure and fluid flow rate settings.

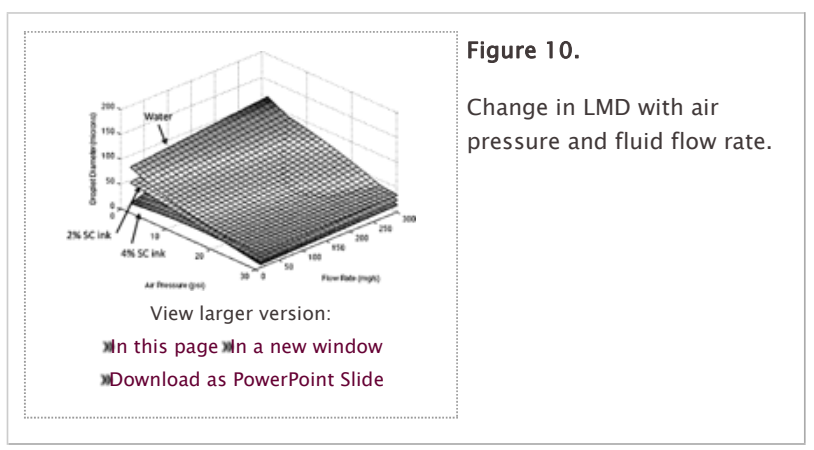

What these results imply is that a fluid of higher viscosity produces smaller droplets. Upon cursory observation, this seems entirely incorrect, as a more viscous fluid should require more energy for atomization. The viscosity of a liquid has been noted, in the literature, to exert a significant influence on the average drop size of a spray, the size decreasing with decreasing viscosity. ${ }^{28}$ There are key differences between the carbon inks and water, however. More than half of the ink is composed of isopropyl alcohol (IPA), which has a much lower boiling point than that of water. It can be reasoned that as the atomization process increases the surface area of the fluid, by creating thousands of tiny droplets, the rate at which the alcohol content is evaporating is increased tremendously, and the droplet loses volume. So at the point at which the measurement is made, an ink droplet could be smaller than a water droplet produced at the same air pressure and flow rate settings.

Another contributing factor to the smaller drop sizes produced by ink is that IPA lowers the surface tension of water. The primary force resisting the drop formation process in a liquid is surface tension. As the surface tension of inks is lowered, it could be hypothesized that they will require less energy for atomization, or, for the same energy, have a higher degree of atomization, as shown in the model.

An observation made is that, in all cases, increasing the air pressure decreases the droplet size. The same trend is observed across all the fluids, starting from water to $4 \%$ SC ink. This result implies that air pressure is a key driver of droplet size, which in turn could possibly affect the electrode microstructure. This observation is consistent with what was expected, as a higher pressure would provide more energy for atomization, thereby producing finer droplets. 
Examining the results in Figure 10 further, it can be observed that as the flow rate is increased, while keeping the pressure constant, the droplet size increases. We propose the following explanation: as the pressure is held constant, the atomizing energy remains the same, but the amount of liquid to be atomized per unit time increases; hence, less energy is transferred to the fluid on a mass basis, producing larger droplets.

The utility of these models for the spraying process of catalyst ink onto substrates during the fabrication of PEMFC and DMFC electrodes is that it allows for a clear identification of what droplet sizes can be expected, and how the spray morphology can be tuned. What influence the spray size has on the electrode structure and its performance is discussed in detail in the following sections.

Spraying pressure/Drop size

The second stage of the optimization process was to ascertain the optimal spraying pressure for the experimental set-up, in particular the Nordson EFD$781 \mathrm{~S}$ nozzle. It was ascertained that higher pressures translated into smaller droplet sizes, but it was necessary to determine how the droplet size would affect microstructure and the overall MEA performance.

The first step in investigating the effects of different catalyst ink droplet sizes on the electrode microstructure was to observe electrode samples produced by these sprays in a SEM. Six samples were prepared, three anodes and three cathodes, sprayed at different pressures, which were mounted and observed in an electron microscope.

Figure 11 shows the results of the SEM study. The anode microstructure is changing as the spray pressure changes, the pore distribution and sizes are clearly affected by the smaller drop sizes. Whereas, there is little change observed on the cathode microstructure.

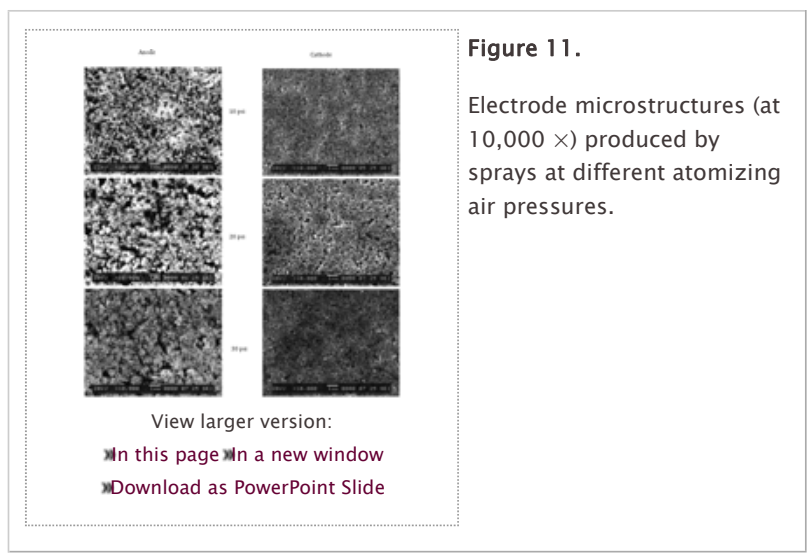

In order to develop a better understanding of the microstructure produced at the $30 \mathrm{psi}$ atomizing air pressure, samples were studied at higher magnification, as shown in Figure 12, which shows an anode and cathode sprayed at $30 \mathrm{psi}$ at high magnification. The anode clearly consists of clumps of catalyst and ionomer particles, between which exist pores. The microstructure appears less sensitive to the atomizing air pressure. From these observations, we believe that smaller droplets produce a much more refined pore structure on the anode, which could potentially allow for better mass transport, and thus increased electronic and protonic conductivity between the different catalyst sites.

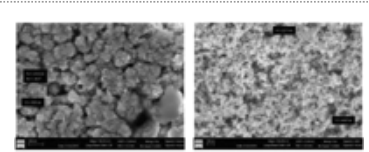

View larger version

wn this page $m$ a new window

wDownload as PowerPoint Slide
Figure 12.

Microstructure of electrode sprayed at 30 psi at 30,000 $\times$ magnification. 
Mass transport could potentially improve because smaller pores, which appear to have a much denser network, replace the larger pores visible in the $10 \mathrm{psi}$ electrodes. Such a structure could potentially impact the $\mathrm{CO}_{2}$ formation and removal process on the catalyst sites.

The next stage in the atomization air pressure optimization process was to determine if the change in microstructure translated into any difference in MEA performance. In order to address this issue, MEAs were prepared with the method described previously. Anode and cathode loadings were maintained at $2.5 \mathrm{mg} / \mathrm{cm}^{2}$ using Pt-Ru Black and Pt- Black respectively. Nafion content was kept at $10 \mathrm{wt}$ \% in both the electrodes. 15 MEA's were prepared, 5 each at 10 , 20 and 30 psi spray pressures. These MEAs represent two different batches of inks, sprayed at different times, but with the same process conditions.

Figure 13 shows the results of this study. As is evident, MEAs sprayed at $30 \mathrm{psi}$ far outperformed those prepared at lower pressures. As was suspected by studying the microstructure, the refined pore network allows for a significant improvement in the overall MEA performance. These results answer a key question posed within this study. There is a clear relation between droplet size of the sprayed catalyst ink and microstructure in the electrode, which in turn affects the overall MEA performance. Higher atomizing air pressures produce smaller droplets, which produce finer pores in the electrode, which improve the MEA electrochemical performance significantly.

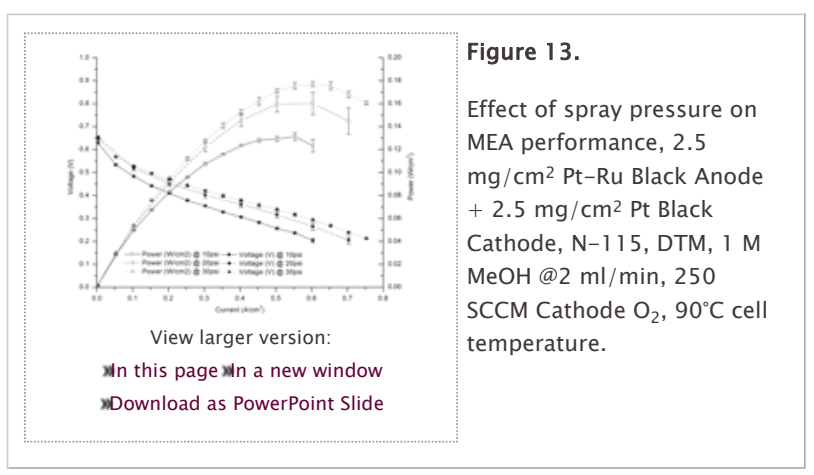

We do find slightly lower apparent open-circuit potentials than in some of the other reported DMFC performance curves in the literature, 23,25,29 but attribute this behavior to an experimental artifact, rather than a flaw in the MEA manufacturing process. Cell resistances were measured as part of the assembly testing process to preclude the possibility of a short; while issues with edge sealing might afford a slightly higher methanol crossover rate than in the literature, we note also that most of the literature data measures currents down to very low current densities so as to ascertain the specific electrocatalyst performance and to measure such parameters as the Tafel slope. In the experiments shown in Figures 13 and 14, test stand limitations restricted the lowest current measured to roughly $10 \mathrm{~mA} / \mathrm{cm}^{2}$, which does not yield the true open-circuit condition. We find similar values between our results and the literature in terms of extrapolation of the linear ohmically controlled region back to the $y$-axis.

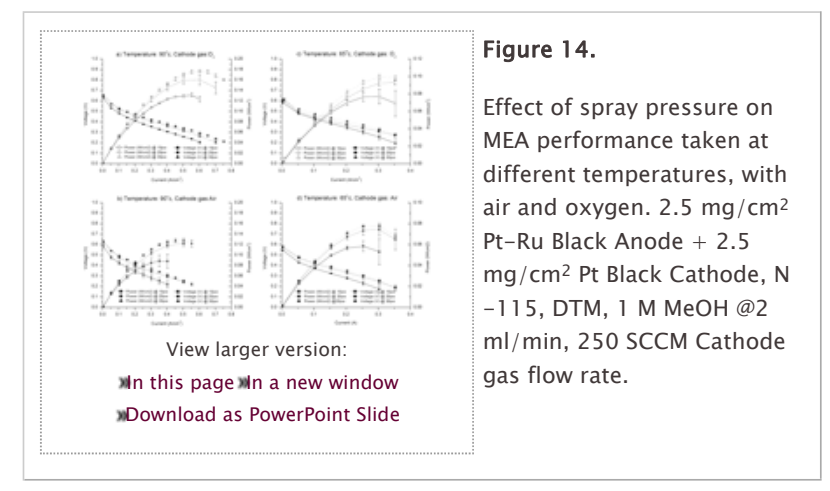

Figure 14 shows the performance of the MEAs at different temperatures, with air and oxygen. As shown, the best performance is with oxygen at $90^{\circ} \mathrm{C}$, with a peak power of approximately $180 \mathrm{~mW} / \mathrm{cm}^{2}$. The performance with air at $90^{\circ} \mathrm{C}$ 
decreases to approximately $130 \mathrm{~mW} / \mathrm{cm}^{2}$. MEA performances at $65^{\circ} \mathrm{C}$ follow the same general trend. When MEA's are tested with oxygen, a peak power of 100 $\mathrm{mw} / \mathrm{cm}^{2}$ was achieved; whereas when air is used, a peak power of $80 \mathrm{mw} / \mathrm{cm}^{2}$ was achieved.

The results are summarized in Figure 15, by tabulating the peak power obtained for each run. At all voltage levels, power increases with increasing spray pressure. 30 psi corresponds to the maximum that was achievable with our laboratory apparatus.

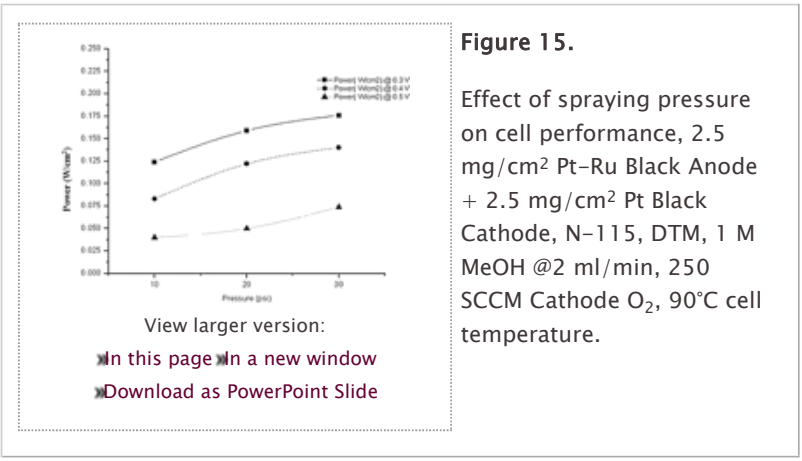

\section{Conclusion}

The effect of the droplet size of an atomized spray of catalyst ink, produced by a two-fluid air assist atomizing nozzle on the electrochemical performance of electrode subsequently produced by this sprayed catalyst ink, is studied in detail. It is shown that varying nozzle control parameters, specifically the fluid flow rate and atomizing air pressure, could produce sprays with significantly different characteristics. In order to quantify and quantitatively differentiate between different sprays, a droplet size measuring apparatus, based on highspeed photography, was fabricated. Using this apparatus, the nozzle performance was characterized with different fluids, and an empirical model was fit to the data to understand its response under different operation conditions.

Based on these characterization studies, sprays with different mean droplet sizes are produced, from which electrodes and MEAs are fabricated. The electrode microstructure was investigated, which revealed that higher atomizing air pressures produced smaller droplets, which positively influenced the electrode structure, producing a finer pore structure. Such a pore structure is reasoned to affect the mass transport of reactants and reaction products in the electrode. Subsequent electrochemical performance testing confirmed these findings, i.e., electrodes fabricated from sprays with smaller droplet sizes performed significantly better.

\section{Acknowledgments}

Financial support by the Office of Naval Research MURI grants No. N00014-071-0758 is gratefully acknowledged. The authors would also like to acknowledge the support provided from the Cullen Endowed Professorship in Engineering, The University of Texas at Austin. Any opinions, findings, or conclusions found in this paper are those of the authors and do not necessarily reflect the views of the sponsors.

\section{Footnotes}

$\downarrow^{*}$ Electrochemical Society Active Member

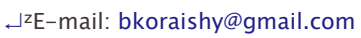

This was Paper 771 presented at the Las Vegas, Nevada Meeting of the Society October 10-15, 2010.

$\downarrow$ Nafion is a registered trademark of E. I. du Pont de Nemours and Company.

Manuscript submitted July 14, 2011. 
Revised manuscript received August 23, 2011.

Published November 1, 2011.

C2011 ECS - The Electrochemical Society

$0013-4651 / 2011 / 158(12) / B 1459 / 13 /$

\section{References}

1. I asher S. Sinha I.. Yana Y., Cost Analyses of Fuel Cell Stack/Systems, in, DOE, Cambridge, MA (2008).

2. Mehta V., Cooper J. S., Journal of Power Sources, 114, 32 (2003). CrossRef

3. Cinrai I .. Antolini F.. Pozio A., Passalacqua E., Electrochimica Acta, 43,

3675 (1998). CrossRef

4. Kim B., Kim J., Cha B. J., Jung B., Journal of Membrane Science, 280, 270

(2006). CrossRef

5. Reshetenko T. V., Kim H. T., Krewer U., Kweon H. J., Fue/ Cells, 7, 238

(2007). CrossRef

6. Sister V. G., Fateev V. N., Bokach D. A., Russian Journal of Electrochemistry, 43, 1097 (2007). CrossRef

7. Tang H., Wang S., Pan M., Jiang S. P., Ruan Y., Electrochimica Acta, 52, 3714 (2007). CrossRef

8. Gulzow E., Reissner R., Weisshaar S., Kaz T., Fuel Cells, 3, 48 (2003). CrossRef

9. Litster S., McLean G., Journal of Power Sources, 130, 61 (2004). CrossRef

10. Lefebvre A. H., Atomization and Sprays, CRC Press (1989).

11. Varaa A. Rrunelli N. A. I nuie M. W.. Cianis K. P., Haile S. M., Journal of Materials Chemistry, 20, 6309 (2010). CrossRef

1). IImeda M. Kawaduchi S. IIchida I. Iananese Inurnal of Annlied Phvsics Part 1-Reaular Papers Brief Communications \& Review Papers, 45, 6049 (2006). CrossRef

13. Nasr G. G.. Yule A. J., Bendig L., Industrial Sprays and Atomization, Springer (2002).

14. Dodge L. G., Appl. Opt., 26, 1328 (1987). CrossRef Medline

15. liana Y.. Ieon H. Y.. Tian L.. Bode L. E., International Journal of Multiphase Flow, 36, 193 (2010). CrossRef

16. Mugele R., Evans H., Industrial \& Engineering Chemistry, 43, 1317 (1951). CrossRef

17. Jackman L. A., Lavelle S. P., Nolan P. F., Journal of Aerosol Science, 21, S563 (1990). CrossRef

18. Dehhi A. Knasiak K. F., Suckow D., Guentay S., Journal of Aerosol Science, 32, 33 (2001). CrossRef

19. Liu H., Science and Engineering of Droplets, William Andrew (2000).

20.I. KLD Labs. KId Labs - Droplet Measurment Technology, in, Huntington Station, NY (2011).

21. Bayvel L., Orzechowski Z., Liquid Atomization, Taylor \& Francis (1993).

22.Imagewarp.com, Imagewarp Image analysis software, in, A \& B Soft.com (2011).

23. Ren X...Wilson M. S. Cinttesfeld S., Journal of The Electrochemical Society, 143, L12 (1996). CrossRef

24. Koraishv B. M.. Morter D.. Solomon S.. McDonald B., Meyers J. P., Wood K., ECS Transactions, 33, 1997 (2010). CrossRef

75. Ren X. Snrincer T. Ciottesfeld S., Journal of The Electrochemical Society, 147, 92 (2000). CrossRef

26. Park I.-S.. Li W., Manthiram A., Journal of Power Sources, 195, 7078

(2010). CrossRef

77. Mnntaomery D. C., Design and Analysis of Experiments, John Wiley \& Sons, Inc (2001).

28. Lavernia E. J., Wu Y., Spray Atomization and Deposition, Wiley (1996).

29. Thomas S. C. Ren X. M., Gottesfeld S., Zelenay P., Electrochimica Acta, 47, 3741 (2002). CrossRef 